IdeAs

Idées d'Amériques

$3 \mid 2012$

L'alimentation dans les Amériques au prisme des sciences sociales

\title{
L'alimentation : enjeux théoriques et empiriques dans les Amériques
}

Theoretical and empirical aspects to food in North and South America

La alimentación: cuestiones teóricas y empíricas en las Américas

\section{Philippe Cardon et Domingo Garcia-Garza}

\section{OpenEdition}

Journals

Édition électronique

URL : https://journals.openedition.org/ideas/403

DOI : $10.4000 /$ ideas.403

ISSN : 1950-5701

Traduction(s) :

Theoretical and empirical aspects to food in North and South America - URL : https:// journals.openedition.org/ideas/2293 [en]

La alimentación: cuestiones teóricas y empíricas en las Américas - URL : https:// journals.openedition.org/ideas/2294 [es]

A alimentação: questões teóricas e empíricas nas Américas - URL : https://journals.openedition.org/ ideas/3139 [pt]

Éditeur

Institut des Amériques

Référence électronique

Philippe Cardon et Domingo Garcia-Garza, «L'alimentation : enjeux théoriques et empiriques dans les Amériques », IdeAs [En ligne], 3 | 2012, mis en ligne le 26 février 2018, consulté le 19 octobre 2022.

URL : http://journals.openedition.org/ideas/403 ; DOI : https://doi.org/10.4000/ideas.403

Ce document a été généré automatiquement le 19 octobre 2022

\section{c) (1) $\ominus$}

Creative Commons - Attribution - Pas d'Utilisation Commerciale - Pas de Modification 4.0 International - CC BY-NC-ND 4.0

https://creativecommons.org/licenses/by-nc-nd/4.0/ 


\title{
L'alimentation : enjeux théoriques et empiriques dans les Amériques
}

\author{
Theoretical and empirical aspects to food in North and South America \\ La alimentación: cuestiones teóricas y empíricas en las Américas
}

Philippe Cardon et Domingo Garcia-Garza

1 L'alimentation n'a été retenue que marginalement pour étudier les sociétés de l'Amérique du Sud. Inversement, aux États-Unis et au Canada, la recherche sur l'alimentation a connu, comme en Europe, un développement plus important. Ce troisième numéro thématique de la revue IdeAs entend combler cet écart et relancer une réflexion longtemps oubliée ou délaissée par les sciences sociales, mais toujours présente aussi bien dans l'imaginaire que dans le sens commun des deux côtés de l'Atlantique. L'autre objectif de ce dossier est de porter un regard croisé sur l'alimentation à l'échelle continentale, dans une optique sociologique, anthropologique et historique.

2 «Fait social total», l'alimentation offre en effet un angle privilégié pour étudier les sociétés. Les historiens et les anthropologues figurent parmi les premiers à s'y être intéressés. Du côté européen, les travaux de Claude Levi-Strauss (1964), Fernand Braudel (1967), ainsi que ceux de Jean-Louis Flandrin et Massimo Montanari (1996) ont ouvert la voie à des chantiers de recherche fructueux. Du côté américain, les historiens (Garnsey, 1999 ; Gabaccia, 1998 ; Bauer, 2001) et les anthropologues (Douglas, 1965 ; Goody, 1982; Mintz, 1996; Mintz et Dubois, 2002) sont devenus des références en la matière. Les recherches menées en Amérique latine, ou celles publiées en espagnol et en portugais sur la question alimentaire ont privilégié une perspective historique (Freyre, 1933 ; Cascudo, 1983 ; Flores, 2003; Fernandez-Armesto, 2004 ; Magalhães, 2004; Bourgues et al., 2009), anthropologique (Vargas, 1993; Ortiz et al., 2004; Contreras et Gracia, 2005; Carrasco, 2007; Bertran, 2010), voire pluridisciplinaire (Oseguera, 2003 ; Long-Solis y Vargas, 2005). Ces études s'intéressent essentiellement aux dimensions folkloriques, symboliques ou identitaires régionales de l'alimentation ou aux pratiques des groupes minoritaires (de Suremain et Katz, 2009). 
Dans ce troisième dossier thématique de la revue IdeAs portant sur l'alimentation dans les sociétés américaines (mondes anglo-saxon et hispanique), les différentes contributions proposent une lecture sociologique, anthropologique et historique du fait alimentaire. Elles portent tout d'abord sur l'alimentation comme marqueur d'identité, qu'il s'agisse d'identité de différents groupes sociaux ou d'identité nationale, voire nationaliste de pays latino-américains ou du nord des Amériques. Elles abordent notamment les enjeux de distinction sociale, l'invention d'une " tradition alimentaire " ou encore la «fabrication » d'une alimentation qui s'inscrit dans un discours national, voire nationaliste. D'autres s'intéressent à la production et à la consommation d'aliments locaux, aux conditions économiques et sociales qu'implique leur mise sur le marché tant à l'échelle locale qu'à l'échelle internationale. Par ailleurs, des contributions analysent le rôle de la diffusion de produits alimentaires entre pays sur les pratiques alimentaires de migrants, notamment du sud vers le nord, ouvrant une réflexion sur les processus de "métissage " qui ont donné lieu aux cuisines latinoaméricaines. Ainsi, si l'alimentation reste un marqueur identitaire fort (de classe, mais aussi nationale), elle joue aussi un rôle dans les processus de métissage alimentaire, d'«hybridation» des pratiques notamment dans le contexte des migrations. À cet égard, la problématique de la diffusion de styles, de modes, de normes alimentaires traverse de manière implicite l'ensemble des textes, qu'il s'agisse de la diffusion de nouvelles normes gastronomiques auprès des élites brésiliennes (Carolina Pulici), de nouvelles normes alimentaires "éthiques" au Canada auprès de différentes populations (Josée Johnston et al.), d'un produit de luxe auprès de l'ensemble d'une population (Natalia Milanesio), des pratiques populaires vers les classes favorisées (Garcia-Garza), du modèle créole en Argentine, à Cuba et au Mexique (Jeffrey Pilcher), de pratiques alimentaires "hybrides» entre États-Unis et pays d'Amérique du Sud (Maximino Matus) ou encore de pratiques états-uniennes auprès de migrants mexicains (Frida Calderon-Bony). Se pose parallèlement la question des conditions sociales d'appropriation de ces nouveaux styles alimentaires par les populations concernées. Un des enjeux de ce dossier thématique est alors de se demander dans quelle mesure les sociologies de l'alimentation, particulièrement riches en France (Poulain, 2001, 2002, 2012 ; Corbeau, 2000; Poulain et Corbeau, 2002; Régnier et al., 2006; Fischler et Masson, 2007), sont transposables aux sociétés du continent américain.

\section{L'alimentation comme facteur de différenciation sociale}

4 De nombreux travaux soulignent combien l'alimentation constitue un marqueur social, qu'il s'agisse de travaux portant sur le poids des dépenses en alimentation sur le niveau de vie au Mexique par exemple (Martinez y Villezca, 2003 ; Torres et Trápaga, 2001) ou sur le rôle de la vente de certains types d'aliments comme activité alternative économique à l'exclusion sociale dans des pays en «voie de développement " (Babb, 1987; Tinker, 1997 ; Chaves, 2006 ; Garcia-Garza, 2009). Ces travaux s'inscrivent plus globalement dans la problématique des différenciations sociales des consommations, qui est par ailleurs bien renseignée dans les travaux européens, tout particulièrement dans la sociologie française (Herpin et Verger, 2008), depuis les premiers travaux de Maurice Halbwachs portant sur les consommations ouvrières et poursuivis par ses 
héritiers, Pierre Bourdieu et Claude Grignon, notamment. Ces derniers ont largement montré l'importance de la diversité sociale des consommations alimentaires qui, audelà de certains plats ou savoir-faire culinaires régionaux, nationaux, patrimoniaux, restent profondément marquées par les effets de structure de par l'appartenance d'un groupe social (Grignon et Grignon, 1980 ; Régnier et al., 2006), l'âge et le cycle de vie (Cardon, 2010, 2009a, 2009b ; Cardon et Gojard, 2009 ; Gojard, 2010) ou encore la région (Poulain, 2001). De plus, l'émergence d'une nouvelle offre alimentaire, liée au développement de l'industrie agroalimentaire, des grandes surfaces, de la restauration rapide et de nouveaux produits (les surgelés, par exemple), n'a pas remis en cause la conservation de certaines "cultures alimentaires locales"(Poulain, 2002). Ainsi l'alimentation reste un marqueur social fort de par les pratiques propres à certains groupes sociaux.

\section{Les enjeux autour de la distinction sociale}

5 L'étude de la structure sociale des consommations ouvre tout d'abord classiquement sur une analyse des mécanismes de la distinction telle que l'a notamment développée Pierre Bourdieu dans son ouvrage majeur La distinction (1979) dans lequel il défend la thèse selon laquelle la position sociale dans l'espace social détermine un style de vie et par extension des goûts, notamment alimentaires socialement marqués (Bourdieu, 1976). Les goûts étant « classants » c'est-à-dire supports de classement, sont aussi des marqueurs de la position sociale dans l'espace social. Dans ce numéro, Johnston, Szabo et Rodney, de même que Garcia-Garza, mettent en évidence que les caractéristiques sociales exercent une influence non négligeable sur la consommation alimentaire. Johnston et al. montrent par exemple la manière dont se construit une alimentation "éthique " au Canada à partir de l'observation de deux groupes sociaux distincts (classes populaires versus classes favorisées). Ces auteurs soulignent le rôle joué par le capital culturel, au-delà de l'origine sociale, dans les motivations des consommateurs à acheter des produits considérés comme «bio » ou respectueux de l'environnement et de la santé. Pour sa part, Garcia-Garza met en lumière comment le processus d'anoblissement de produits populaires contribue à redéfinir la légitimité de la hiérarchie alimentaire au Mexique.

6 Par ailleurs, différents textes dans ce numéro démontrent que de nouveaux styles d'alimentation ne font pas forcément disparaître les différences sociales ni les rapports symboliques entre classes. De fait, les nouvelles habitudes alimentaires tracent autant qu'avant les frontières symboliques entre les différents groupes sociaux (Bourdieu, 1979 ; Cole, 2008). Lamont (1992) a par exemple montré que les frontières, classées en trois catégories (socio-économique, culturelle, morale), peuvent intégrer ou exclure les membres d'une société selon leur statut, la possession de ressources, leurs avancées sociales. Elle a montré la corrélation des styles de vie avec les différents statuts sociaux. Cet aspect a déjà été soulevé par Bourdieu (1979) lorsqu'il a étudié la dimension culturelle dans les jeux de différenciation sociale.

7 Dans tous les cas, le maintien des différenciations sociales indépendamment de la diffusion de nouveaux styles alimentaires de part et d'autre de l'échelle sociale, relance le débat sur l'omnivorisme culturel (Peterson, 1992; Peterson et Kern, 1996; Duval, 2010; Coulangeon, 2011). Comme n'importe quelle autre pratique culturelle, l'alimentation obéit aux jeux de différenciation sociale en fonction des positions des 
individus et des groupes dans l'espace social, et ce particulièrement en fonction du niveau d'instruction et des origines socioculturelles. Les pratiques culturelles correspondent selon ce schéma aux différentes classes sociales. Le comportement omnivore ne s'observe que dans les groupes favorisés, et ce lorsque ces derniers présentent un "éclectisme éclairé " à la culture, c'est-à-dire une diversification culturelle sélective (Donnat, 1994 ; Eloy et Paletta, 2008 ; Eloy, 2012). Autrement dit, les pratiques culturelles associées à un comportement éclectique ne sont présentes que chez les groupes sociaux favorisés. Par ailleurs, ces pratiques ne se font que de manière ponctuelle, les groupes sociaux favorisés préférant des pratiques culturelles propres à leur milieu d'origine. Qui plus est, et comme Johnston et Bauman (2007: 198) le soulignent, Bourdieu avait déjà repéré l'émergence de tendances à l'omnivorisme chez les individus plus riches en capital culturel qu'en capital économique (les professeurs en l'occurrence) qui de ce fait ont des consommations ascétiques et recherchent l'«exotisme» et le "populisme culinaire» (Bourdieu, 1979: 207). À partir de différentes contributions dans ce numéro IdeAs sur l'alimentation dans les Amériques, on peut mesurer la force et la validité de la théorie de la légitimité culturelle esquissée par Bourdieu dès la fin des années 1970.

\section{L'appropriation de nouveaux modèles alimentaires}

8 Un des enjeux théoriques de ce numéro IdeAs est dès lors de rendre compte de l'appropriation sociale de nouveaux modèles alimentaires, qu'il s'agisse de nouvelles gastronomies telles que le propose Carolina Pulici dans son étude des élites brésiliennes (banquiers, ambassadeurs, collectionneurs d'art, rentiers, industriels, entrepreneurs) ou d'une alimentation éthique étudiée par Josée Johnston et ses collègues au Canada. Ces auteurs cherchent à comprendre en quoi et à quelles conditions sociales ces nouveaux modèles deviennent-ils ou non des supports de distinction sociale. Caroline Pulici s'attache ainsi à analyser (renouant ici avec une méthodologie de recherche initiée par Norbert Elias dans son ouvrage La Civilisation des mours) des manuels de savoir-vivre récemment publiés au Brésil et des critiques gastronomiques parues dans la presse pauliste entre 2005 et 2009 pour mieux en dégager certaines normes qui s'imposent aujourd'hui comme légitimes en matière de cuisine et de manières de table. L'auteure montre comment les élites se réapproprient ou non ces nouvelles normes alimentaires dans les représentations sociales d'elles-mêmes et dans leurs rapports aux autres groupes sociaux, et comment ces nouveaux modèles alimentaires permettent aux élites de se situer socialement par rapport aux autres, en particulier aux classes populaires. Ainsi, à une alimentation populaire jugée "vorace " par les élites (la "voracité populaire» serait l'expression des sentiments naturels), ces dernières opposent une culture alimentaire qui rejette précisément ces traits de perception propres aux classes populaires. Cette censure des sentiments naturels fonctionne comme règle de modération alimentaire, de retenue et de sens esthétique qui caractérise alors un style bourgeois par rapport à la nourriture. L'appropriation de cette nouvelle cuisine gastronomique maintient la distinction sociale en ce dont elle participe à l'élaboration d'une représentation et d'une éthique reposant sur le contrôle de soi. L'auteur, qui reprend ici à son compte la thèse du contrôle de soi développée par Norbert Elias à propos des sociétés européennes, écrit : «si le 'peuple', qui a moins de choses à attendre de l'avenir, se perd dans les sensualismes, l'élite fait preuve de la maitrise de soi dans toutes les directions, c'est-à-dire, dans le contrôle de ses appétits 
corporels, mais aussi de ses conditions idéales et matérielles d'existence ». Ici, l'alimentation, parce qu'elle est liée à une éthique de vie propre à un groupe, permet de maintenir, de marquer la distance avec d'autres groupes sociaux et donc de les différencier. On comprend alors combien ces nouvelles normes gastronomiques sont d'autant plus aisément appropriées par l'élite brésilienne qu'elles fonctionnent comme un répertoire symbolique permettant de distinguer, de mettre à distance les autres groupes sociaux au regard des différences dans la maîtrise de soi.

Pour autant, ce qui a priori distingue différencie-t-il toujours "autant"? C'est en substance la question traitée par Josée Johnston et al. à propos de la diffusion de l'alimentation dite "éthique» dans la société canadienne : modèle alimentaire fortement valorisé par les pouvoirs publics dès lors qu'il représente un nouveau marché potentiel et est censé apporter une sorte de plus-value sanitaire. Sur la base d'une enquête qualitative (40 familles), les auteurs cherchent à voir si l'alimentation éthique, qui en principe ressortirait de pratiques culturelles propres aux classes aisées, peut être aussi l'apanage d'autres groupes sociaux, notamment populaires. Autrement dit, elles cherchent à déterminer comment le discours sur l'alimentation éthique est perçu et mis en pratique par des consommateurs ne jouissant pas tous des mêmes privilèges. Elles distinguent deux temps dans leur analyse. D'une part, elles cherchent à voir s'il existe ou non des différences de classes dans la manière d'appréhender symboliquement l'alimentation éthique, et si cela fait sens pour les individus de différentes catégories sociales. Elles étudient la façon dont les discours sociaux modèlent les pratiques alimentaires. Pour cela, elles ont recours tout d'abord au concept de «répertoire culturel» (Lamont, 1992; Tilly, 1993; Swidler, 1986, 2001) emprunté à la sociologie de la culture (elles développent le concept de « répertoire de l'alimentation éthique ") qui permet de rendre compte de la manière "créative dont les acteurs emploient au quotidien des éléments choisis de ce discours » sur l'éthique. Elles mobilisent ensuite le concept de «frontières symboliques » qui conduit les individus à tracer les limites pour se distinguer soi-même et signifier aussi son appartenance au groupe, s'inscrivant ainsi dans la continuité des travaux de Durkheim et Mauss (1903), Becker (1963), Douglas (1966). Mise à l'épreuve des discours d'individus appartenant à différents milieux sociaux, cette double entrée conceptuelle leur permet de montrer que le privilège économique et culturel favorise l'accès au répertoire dominant de l'alimentation éthique, puisque les personnes issues de milieux sociodémographiques et ethniques populaires, voire marginalisés, ont moins accès à ce répertoire. À l'inverse, ce sont les personnes blanches issues des classes moyennes qui investissent davantage le répertoire de l'alimentation éthique. Pour autant, l'étude des pratiques quotidiennes autour de l'alimentation montre dans un second temps des écarts possibles entre la reconnaissance légitime d'un discours éthique sur l'alimentation et la mise en pratique. D'un côté, les privilèges culturels et économiques, certes, autorisent l'accès au répertoire dominant, mais « ils ne garantissent pas un degré élevé d'implication » dans les pratiques. Pour un grand nombre de personnes issues des milieux privilégiés, indépendamment du fait d'être impliquées ou non dans l'alimentation éthique, ce sont surtout des comportements globaux alimentaires jugés « sains » qui jouent comme un marqueur, et par extension comme facteur de distinction culturelle. A contrario, le fait que les groupes marginalisés s'impliquent moins dans l'alimentation éthique ne signifie pas pour autant «qu'ils ne se sentent pas concernés par les dilemmes moraux liés aux choix alimentaires ». Pour les auteurs, ce résultat est central, car il vient confirmer les travaux qui réfutent la thèse de l'insuffisance morale des classes populaires selon 
laquelle ces dernières ne connaissent pas les enjeux moraux liés à l'alimentation, voire ne s'en soucient guère ou ne s'y impliquent pas (Johnston, Szabo et Rodney).

\section{L'alimentation comme facteur d'identité nationale, voire nationaliste}

10 Si certains goûts alimentaires restent de forts marqueurs sociaux du fait qu'ils différencient les classes sociales, d'autres acquièrent le statut de «patrimoine national ", de bien commun et deviennent des emblèmes, des marqueurs culturels qui transcendent les différences de classes. On peut ainsi étudier l'alimentation comme vecteur d'identité (Pilcher, 1998 ; Thiesse, 1999 ; Bruegel et Lauriaux, 2002). À l'instar de recherches européennes (Poulain, 1997, 2000, 2005 ; Espeitx, 2008; Bessière et Tibère, 2010), certains chercheurs ont, de l'autre côté de l'Atlantique, travaillé sur la patrimonialisation des cuisines latino-américaines, les pratiques alimentaires étant envisagées comme «patrimoine culturel » support d'identité nationale (Florescano, 1993; Álvarez, 2002; Cottom, 2004, 2007; Álvarez y Medina, 2008; Moncusí et Santamarina, 2008 ; Zapata, 2008), même si la recherche dans ce domaine en Amérique latine est encore à l'état embryonnaire (Bak-Geller et Katz, 2011).

11 La célébration du bicentenaire des indépendances latino-américaines était une excellente occasion de réfléchir à la place occupée par l'alimentation dans les sociétés sud-américaines. À cette occasion, on a vu célébrer les plats nationaux des différents pays. Mais très peu d'études prennent suffisamment de recul pour voir la façon dont ces plats contribuent justement à définir l'identité nationale des pays latino-américains à l'aube du XXI ${ }^{e}$ siècle. Or, il est intéressant de voir la manière dont l'identité nationale se construit à la lumière du passé et, dans une plus grande mesure, par rapport à l'étranger. Du nord au sud, les différentes formes d'alimentation pour définir les cuisines nationales gardent un rapport, soit de rejet soit d'attraction, à l'égard de l'international. Le Mexique par exemple ne cesse de faire appel à son passé précolombien pour construire la " cuisine mexicaine ", même si cette dernière n'a pas vraiment existé comme telle avant le $\mathrm{XX}^{\mathrm{e}}$ siècle dans la mesure où elle est née de la fusion de divers éléments et techniques à la fois exogènes et endogènes (Pilcher, 1998). À l'opposé, l'Argentine rejette ce passé et ne se définit que par des liens très forts avec l'étranger, dus évidemment aux migrations européennes récentes. Ce qui se traduit par un certain cosmopolitisme de la cuisine argentine (Pilcher, 2012), du moins dans la région connue comme La Pampa (Milanesio).

\section{D'une diffusion du haut vers le bas....}

Dans certains cas, ce bien commun national, voire patrimonial comme construction sociale, devient parfois le support, l'outil d'une propagande nationaliste et objet de propagande politique. L'alimentation peut ainsi servir à rassembler un peuple dès lors qu'elle est érigée en emblème national, support d'une politique idéologique forte à visée nationaliste. C'est ce que montre précisément Natalia Milanesio dans son étude du rôle joué par la production et la consommation de bœuf dans la propagande " péroniste " (du nom de l'ancien président argentin, Juan Domingo Perón). Dans son examen des aspects structurels et institutionnels caractéristiques de la politique alimentaire péroniste des années 1950, elle mobilise le concept de «virtuosité 
sémiotique » de l'alimentation développé par l'anthropologue Arjun Appadurai (1988), c'est-à-dire la capacité du signe culturel à pouvoir transmettre des messages multiples, pour mieux mettre au jour les rôles et les utilisations idéologiques que fait l'État de la consommation alimentaire, et particulièrement les implications symboliques de la consommation de bœuf dans sa politique alimentaire. Comme elle le souligne en conclusion, «la politique bovine du péronisme d'avant 1950 dépass[e] la simple augmentation de la consommation par personne: elle redéfinit l'image de l'Argentine au sein de l'économie mondiale, valid[e] le concept d'autodétermination économique et remet en question, du moins au niveau du discours, le rôle du capitalisme international ». Traditionnellement considérée comme une nourriture de luxe, la viande devait être le symbole de l'accès des classes populaires à de nouveaux biens et donc à une nouvelle place dans la société. La politique péroniste a ainsi contribué à diffuser la consommation de viande à des fins politiques : préférer les consommateurs nationaux aux marchés extérieurs pour favoriser la diffusion d'une puissante idéologie de souveraineté économique via la politique bovine. Outre les enjeux politiques et électoralistes (dont certains sont associés au populisme), l'érection du bœuf comme emblème national a servi à redéfinir les rôles traditionnels masculin et féminin: «traditionnellement cuisinés par des femmes, les plats recommandés par le gouvernement péroniste réaffirmaient le rôle essentiel de la femme dans la construction de l'identité culinaire nationale, un mouvement éloigné de la figure de l'asador mâle ». Car traditionnellement les hommes n'interviennent dans la cuisine que dans la préparation de la viande grillée.

Par cet exemple, on comprend bien comment la diffusion verticale vers les classes populaires de certains mets, marqueurs indiscutables de classe sociale car réservés généralement aux élites, permet d'asseoir une politique générale nationaliste. Or, la question de la diffusion des goûts occupe une place centrale dans la sociologie de l'alimentation, car elle oblige à penser l'espace et la différenciation sociale de manière dynamique et non figée. Certains groupes sociaux empruntent à d'autres certaines de leurs spécificités. D'autres encore, au nom d'un principe politique, comme nous venons de le voir dans l'exemple de la politique péroniste, travaillent à diffuser leur modèle alimentaire. Mais la diffusion des modèles et des goûts alimentaires transcende aussi les frontières symboliques entre les classes (Milanesio; Garcia-Garza; Pilcher; Johnston et Bauman, 2010) et l'on comprend ainsi combien l'alimentation est loin d'être anodine et encore moins neutre politiquement. A partir du moment où l'alimentation concerne tous les individus et que l'État est chargé d'en assurer les conditions d'accès, ainsi que d'en garantir une certaine qualité (Stanziani, 2005) pour préserver la santé de la population, elle devient un enjeu politique.

\section{... à une diffusion du bas vers le haut}

Mais cette diffusion verticale se fait aussi du bas vers le haut. C'est ce que Domingo Garcia-Garza démontre dans son article sur les pratiques populaires à partir du cas des tacos (galettes salées farcies) dans la ville de Monterrey au Mexique. Au départ perçus comme un produit populaire, les tacos se sont répandus dans tout l'espace social à partir des années 1990, dans la mesure où ils se sont adaptés aux goûts et aux modalités de consommation des différents milieux, particulièrement les plus aisés. Bien que leur consommation se soit généralisée dans l'ensemble de la société, les écarts qui différencient les groupes sociaux se maintiennent. Garcia-Garza démontre que cet 
«embourgeoisement» des plats et pratiques populaires n'a été possible qu'au prix d'une montée en gamme et d'une recontextualisation dans un cadre plus conforme aux canons de la restauration classique. Cette opération a été possible dans la mesure où l'image des tacos ainsi revalorisée contribue à définir l'identité nationale. Mais cette contribution s'explique aussi par le fait que ces pratiques renvoient à la notion de terroir et d'authenticité, notamment quand on utilise des produits considérés aujourd'hui comme "gourmets». Produits jadis dévalorisés, les tacos d'insectes, de sauterelles ou d'huitlacoche (champignon qui pousse sur le maïs, considéré comme l'équivalent du caviar au Mexique), sont devenus aujourd'hui une source de fierté nationale et de distinction sociale, principalement pour les élites renvoie au même temps à redéfinir la légitimité des plats populaires et par conséquent, la hiérarchie des aliments au Mexique.

\section{Diffusion et métamorphoses des goûts entre pays}

Si les cas étudiés jusqu'à présent posaient de manière plus ou moins explicite la question de la diffusion et de la circulation des modes alimentaires entre groupes sociaux dans un contexte national, cette question se pose de manière explicite dans le cas de la circulation transfrontalière des biens alimentaires. Cette problématique a fait l'objet de nombreux débats tant en Europe qu'aux États-Unis notamment au sujet de la diffusion de modèles alimentaires à l'échelle planétaire et de leur appropriation ou non par les cultures nationales. Ainsi, à la thèse de la «mac-donaldisation » de la société élaborée au début des années 1990 (Ritzer, 1993), aujourd'hui largement critiquée, s'oppose une thèse de l'« ère du sur-mesure de masse » (Taylor, Smith et Lyon, 1998) : la diffusion de modes alimentaires extranationaux nécessite une certaine conformité aux habitudes locales de consommation. Dans cette perspective, c'est la dimension nationale, c'est-à-dire locale, qui sert de point d'observation: le chercheur tente de comprendre comment des styles alimentaires extranationaux importés sont appropriés ou non à l'échelle d'un territoire national.

\section{Le poids du contexte national dans la diffusion de modèles internationaux}

Un des enjeux est tout d'abord de comprendre comment un espace national gère à la fois des traditions locales et nationales dans un contexte de forte diffusion, à l'échelle mondiale, de pratiques transnationales. Cet enjeu du rapport entre local et global est précisément l'objet de l'article de Jeffrey Pilcher sur trois pays latino-américains (Argentine, Cuba et Mexique). Dans une perspective historique, il étudie les interactions entre pratiques locales et internationales, et montre combien le local et le global restent fortement imbriqués; mais cette imbrication varie selon les contextes nationaux. Les pratiques culinaires criollas (celles développées dans les nouvelles républiques nées de la fragmentation de l'Empire espagnol), sont plus ou moins affirmées en fonction des ressources disponibles, de l'ancienneté en tant que pays indépendant, du rapport à l'international, et enfin des enjeux politiques internes à chaque pays. Manger "créole» est plus affirmé à Cuba et au Mexique (bien que le terme a été banni du vocabulaire) qu'en Argentine. 
17 Les travaux entamés par Pilcher depuis une dizaine d'années, plus ou moins méconnus en France, montrent que les cuisines et les identités nationales se définissent par rapport aux principaux modèles culinaires incarnés par les pays qui ont servi de modèle de société pour l'Amérique latine (en l'occurrence la France, l'Espagne et l'Angleterre). Il suggère que manger "à la criolla » signifie en définitive adapter les plats européens aux ingrédients disponibles et aux humeurs locales. Mais cela ne se fait ni de la même manière ni avec les mêmes ingrédients. D'autant moins que le rapport que les trois pays gardent avec les anciens empires coloniaux n'est pas le même d'un pays à l'autre. L'intérêt d'une telle approche comparative entre différentes nations est de montrer combien les mécanismes de diffusion et d'intégration de nouveaux aliments, plats ou styles alimentaires, du global au local, doivent s'analyser au regard des contextes nationaux et de la permanence de certaines spécificités locales. Il démontre la manière dont trois traditions culinaires, loin de s'opposer à la globalisation, combinent à la fois le patriotisme et le cosmopolitisme tout en créant les nouvelles cuisines nationales ou criollas. Les cuisines du Mexique, de Cuba et d'Argentine sont emblématiques, chacune à leur manière, de ce processus de syncrétisme culinaire qui s'est opéré à la fin du XIXe siècle. Étudiant la diffusion du modèle criollo dans la littérature et les pratiques culinaires en Argentine, à Cuba et au Mexique, Pilcher montre l'articulation entre ces deux niveaux. Si à Cuba le terme criollo a fini par englober les produits alimentaires locaux autour de la catégorie de viandas (des tubercules comme le manioc, la pomme de terre, la patate douce, etc.), en Argentine, le terme criollo englobera davantage de produits locaux (allant par exemple des spécialités hispaniques empanadas et pucheros aux soupes de squash et au maïs indigène). A contrario, le Mexique n'a pas vraiment intégré le terme criollo(a). Il a développé ces mêmes pratiques culinaires, qu'il a adaptées au climat politique de l'époque et aux goûts locaux. La cuisine criolla a toujours existé au Mexique. Mais elle ne reçoit pas cette appellation du fait que le mot criollo a gardé son ancienne acception (désignant les Espagnols nés au Mexique), trop connotée et donc mal acceptée par les Mexicains fortement imprégnés de nationalisme. Le Mexique a donc évacué l'origine et l'influence espagnoles de la cuisine mexicaine en éliminant les mots pour s'y référer. Les élites mexicaines, qui sont par ailleurs pour la plupart d'origine européenne, se réclament héritières des Aztèques et de leur patrimoine culinaire. Elles revendiquent néanmoins la cuisine mexicaine et ses sources précolombiennes, tout en excluant et marginalisant les peuples indigènes dans la société contemporaine. Elles se sont donc approprié le passé indigène pour forger une cuisine nationale et s'inviter dans le processus d'occidentalisation. Cela s'est fait au prix de la séparation de la cuisine criolla des indigènes qui l'ont créée et de l'effacement des traces européennes. Tout se passe comme s'il fallait se débarrasser de l'origine étrangère des pratiques culinaires pour forger un caractère national. C'est en quelque sorte une manière de couper avec un (douloureux) passé pour inventer un présent plus conforme aux idéaux nationalistes nés après la Révolution (1910-20).

\section{L'alimentation face à la migration}

À cette perspective sociohistorique peuvent se combiner des approches davantage ethnographiques qui s'attachent plutôt à analyser les mécanismes de circulation des biens alimentaires dans la migration entre pays, notamment des pays d'Amérique latine vers les États-Unis. Une des questions majeures que nous retiendrons et qui sous- 
tend les deux articles ici présentés est de savoir à quelles conditions sociales les migrants des pays d'Amérique du Sud vers les États-Unis maintiennent les habitudes alimentaires de leur pays d'origine ou en développent d'autres. Il ne s'agit seulement de déchiffrer comment des modèles extraterritoriaux sont appropriés sur un territoire national, mais de comprendre comment et à quelles conditions les habitudes locales se pérennisent-elles dans une logique de mobilité internationale. Plusieurs éléments de réponse peuvent être mis en avant.

19

20 1. Un des premiers éléments de réponse se trouve précisément dans ce qui se joue, du point de vue identitaire, dans la circulation des biens alimentaires. Dans les deux exemples présentés ici, l'aliment joue comme une "force de rappel identitaire » qui transcende en quelque sorte l'aliment en soi. Ainsi, dans son article, Maximino Matus interroge les stratégies employées par des collectifs de distributeurs et des marchands afin de faire circuler clandestinement des aliments entre les États-Unis et divers pays d'Amérique latine (et inversement) et ce, pour satisfaire et répondre à la «nostalgia de un migrante por el sabor, olor y textura de un alimento». Ainsi, s'il est fréquent de rencontrer aux États-Unis des commerçants et des restaurants qui vendent des produits et des aliments de pays d'Amérique latine, autrement dit des pays d'origine des migrants, il est également courant de trouver dans ces pays des commerçants et des restaurants dont les produits proviennent d'autres pays du monde. Matus se propose alors de dessiner ce qu'il appelle des "paysages alimentaires hybrides » : selon lui, la diversité des formes de migration à l'intérieur des pays d'Amérique latine et entre l'Amérique latine et les États-Unis, fait émerger des hybridations des pratiques alimentaires des migrants qui affectent, non seulement les migrants dans la migration, mais également le pays d'origine. L'exemple de la commune de Oxcutzcab, au sud-est de Mexico est exemplaire à cet égard: on y trouve des restaurants asiatiques, italiens, français et états-uniens. Selon l'auteur, le développement de ces offres alimentaires " hybrides" participe d'une demande des migrants, liée à une certaine nostalgie de nouvelles habitudes alimentaires développées lors des migrations, notamment aux États-Unis. C'est précisément ce que Calderón-Bony étudie : la nostalgie des habitudes alimentaires, créée par les migrants installés aux États-Unis originaires de la commune de Patamban au Mexique. Dans son travail, elle cherche à comprendre où se trouve la place de l'alimentation dans la dynamique identitaire liée à la migration. Elle souligne ainsi combien les migrants sont là aussi fortement attachés à certaines habitudes alimentaires. Les biens alimentaires deviennent alors des produits qui circulent entre leur pays d'origine et leur nouveau pays d'accueil (en sens inverse d'autres biens qu'ils envoient à leur famille dans leur pays d'origine). Or, un des constats empiriques faits par l'auteur tient à la nature même de la justification de cet attachement par les migrants à certains plats liés à leur origine : la nostalgie (Camou, 1994 ; Pilcher, 2012).

2. Le second élément est lié au fait que se développe une "offre » alimentaire du pays d'origine et des marchés de produits alimentaires issus des pays du sud vers les ÉtatsUnis. À cet égard, Calderón-Bony analyse le "marché de la nostalgie » au sens de Hirai (2009) dans le contexte de la migration mexicaine aux États-Unis. Le «marché de la nostalgie » est une notion à partir de laquelle " le migrant cherche à définir le lien qui existe entre l'ouverture d'un commerce avec des produits mexicains comme activité économique réalisée par un migrant et l'offre de produits qui servent d'identification à

IdeAs, 3 | 2012 
la culture mexicaine ", qu'il s'agisse d'épiceries (consacrées uniquement à la vente de produits comestibles tels que boîtes de conserve de différentes sortes de piments, grains de maïs pour préparer le pozole, tortillas et tostadas, feuilles de maïs pour la préparation des tamales, fromage frais) ou de commerces plus importants qui s'apparentent davantage au supermarché. La migration devient donc le support de l'émergence d'une nouvelle offre alimentaire sur le territoire d'accueil des migrants mexicains. Mathus fait le même constat, mais montre qu'en parallèle à cette offre, se développent des «marchés clandestins ». En effet, si la circulation des aliments semble se faire entre pays du sud pour alimenter un marché à forte demande, cela est moins évident pour les produits à destination des États-Unis, tant sont fortes les contraintes sanitaires imposées aux produits qui entrent sur le territoire américain. Dans la mesure où cette demande de produits alimentaires issus des pays d'origine des migrants est forte, l'auteur interroge la manière dont les acteurs (notamment les commerçants) peuvent contourner ce qu'il nomme lui-même des "políticas persecutorias" nordaméricaines qui cherchent à exclure de leur continent certains produits. Il montre ainsi l'émergence d'un marché clandestin diffusant certains produits alimentaires (par exemple d'Oaxaca à Los Angeles en passant par Tijuana). Le développement de ces marchés clandestins semble en partie satisfaire la nostalgie alimentaire de migrants, que ces derniers soient installés ou non aux États-Unis.

3. Pour autant, le fait de disposer de commerces sur place n'exclut pas que les migrants reçoivent des aliments de leur famille restée au pays. Ainsi, outre le développement de marchés officiels et de marchés qui le sont moins, il existe une circulation que nous pouvons qualifier de " privée » car inscrite au cœur même des réseaux familiaux. C'est par exemple ce que démontre parfaitement Calderón-Bony à propos de la circulation des biens alimentaires. L'auteur souligne tout d'abord que les aliments circulent en sens inverse (du pays d'origine au pays d'accueil) que d'autres biens tels que l'argent (du pays d'accueil vers le pays d'origine). D'autre part, comme elle le dit elle-même, « la circulation des aliments entre le village et les États-Unis permet de nourrir des proximités tout en consolidant des liens (familiaux et amicaux) en même temps que ces aliments aident à 'digérer' les distances ». Point central s'il en est, car au fond, on retrouve l'idée du mana proposée par Marcel Mauss (1924), qui soulignait combien, dans l'échange, avec la chose circule l'« esprit de la chose ». C'est à ce titre d'ailleurs qu'on peut comprendre que le «plat d'ici » n'est jamais tout à fait le même que celui de « là-bas ». Car, ce qui circule, plus que l'aliment, c'est l'esprit lié à l'aliment, celui de la famille. Ici, l'échange permet de maintenir l'attachement du migrant à ses compatriotes. La nostalgie est-elle l'esprit même de la chose, du mana ? Ou encore, pour le dire autrement: nous ne mangeons pas les aliments parce que nous en avons la nostalgie, mais nous ressentons de la nostalgie parce que nous les mangeons.

4. Enfin, le dernier élément de réponse a trait au fait que de nouvelles habitudes issues du territoire de migration sont intégrées, permettant ainsi un maintien identitaire entre ici et là-bas. Si cette perspective est présente de manière plus ou moins implicite dans le texte de Mathus à travers son concept d'alimentation hybride (qui présuppose bien la juxtaposition de modes alimentaires par intégration multiple liée au parcours migratoire), elle est centrale dans l'analyse de la place de l'alimentation dans le texte de Calderón-Bony. En effet, cette dernière montre qu'il y a aussi, pour les migrants étudiés, appropriation de la culture alimentaire américaine. Il ne s'agit pas d'opposer « maintien » d'habitudes liées au pays d'origine et « changement » lié au pays d'accueil, mais de les penser de manière dialectique et dynamique comme support d'une 
dynamique identitaire. Ici, l'alimentation sert de support identitaire dans la migration en tant qu'elle permet tout à la fois de maintenir le lien avec son pays, sa région, son village, sa famille et d'intégrer de nouvelles valeurs liées à la culture d'accueil. S'il ne s'agit nullement de revisiter la problématique de l'intégration/assimilation, l'intérêt du texte réside dans le fait de montrer combien l'identité est bien un processus dynamique auquel l'alimentation participe. Or, dès lors que l'auteur souligne cette dimension dynamique du processus identitaire à travers l'alimentation par intégration de nouveaux modes alimentaires propres au pays d'accueil, elle interroge classiquement les enjeux autour de la diffusion de modèles alimentaires au sein d'une société. L'intérêt de son travail est alors d'intégrer une dimension générationnelle dans la compréhension du processus de diffusion des modèles alimentaires américains dans la famille migrante. Calderón-Bony montre ainsi que ce sont les enfants qui sont les vecteurs et les diffuseurs de nouvelles habitudes alimentaires qui viennent, non pas concurrencer les habitudes d'origine, mais les compléter, voire les redessiner.

$\mathrm{Au}$ final, l'ensemble des contributions de ce troisième numéro montre le foisonnement des problématiques et des questionnements aujourd'hui à l'œuvre dans les sciences sociales qui étudient l'alimentation dans les Amériques. Elles soulignent tout l'intérêt heuristique des enjeux théoriques et empiriques qui contribuent aux dialogues et échanges entre disciplines d'une part, et entre pays et continents, d'autre part. Elles invitent à poursuivre ce dialogue Nord/Sud avec d'autres pays, sur d'autres objets à l'instar de la mobilité, de la circulation et de la migration qui sont au cœur même de l'histoire des sociétés humaines.

\section{BIBLIOGRAPHIE}

ÁLVAREZ Marcelo, «El gusto es nuestro. Modelos alimentarios y políticas de patrimonialización », Catauro : Revista Cubana de Antropología, nº 5, 2002, pp. 61-78.

ÁLVAREZ Marcelo y MEDINA Francisco-Xavier (eds.), Identidades en el plato. El patrimonio cultural alimentario entre América y Europa, Barcelona, Icaria, 2008.

APPADURAI Arjun, « How to Make National Cuisine : Cookbooks in Contemporary India », Comparative Studies in Society and History, vol. 30, $\mathrm{n}^{\circ}$ 1, 1988, pp. 3-24.

BABB Florence, « From the Field to the Cooking Pot : Economic crisis and the threat to marketers in Peru », Ethnology, vol. 26, n. 2, 1987, pp. 137-49.

BAK-GELLER Sarah et KATZ Esther, « Semana de Estudios de Cocina y Alimentacion en América Latina », Guadalajara (México), Congreso Internacional : De los primeros recetarios nacionales a las cocinas patrimoniales. 200 años de nacionalismo culinario en América Latina, 12-15 sept., 2011.

BAUER Arnold, Good, Power, History. Latin America's Material Culture, Cambridge, Cambridge University Press, 2001. 
BAUMAN Shyon and JOHNSTON Josée, « Democracy versus Distinction : A Study of Omnivorousness in Gourmet Food Writing ", American Journal of Sociology, vol. 13, n 1, 2007, pp. 165-204.

BECKER Howard, Outsiders : Studies in the Sociology of Deviance, New York, The Free Press, 1963.

BERTRAN Miriam, « Acercamiento antropológico de la alimentación y salud en México », Phycis : Revista de Saúde Coletiva, vol. 20, n² 2, 2010, pp. 387-411.

BESSIÈRE Jacinthe et TIBÈRE Laurence, « Éditorial : Patrimoines alimentaires », Anthropology of Food, $\mathrm{n}^{\circ}$ 8, 15 juin, 2010. Disponible en ligne : http://aof.revues.org/6782.

BOURDIEU Pierre, La distinction. Critique sociale du jugement, Paris, Minuit, 1979.

BOURDIEU Pierre et SAINT MARTIN (de) Monique, "Anatomie du goût ", Actes de la recherche en sciences sociales, $\mathrm{n}^{\circ} 5,1976$, pp. 2-81.

BOURGUES Héctor, BENGOA José y O'DONNELL Alejandro (eds.), Historias de la Nutrición en América Latina, México, SLAN, 2009.

BRAUDEL Fernand, Civilisation matérielle, économie et capitalisme (XV-XVIII siècles), Paris, Armand Colin, 1967.

BRUEGEL Martin et LAURIAUX Bruno (eds.), Histoire et identités alimentaires en Europe, Paris, Hachette, 2002.

CAMOU Ernesto, « La nostalgia del rancho : Notas sobre la cultura urbana y a la carne asada » in Shoko DOODE y Emma-Paulina PÉREZ (eds), Sociedad, economía y cultura alimentaria, Hermosillo, CIAD/CIESAS, 1994, pp. 421-429.

CARDON Philippe, «Regard sociologique sur les pratiques alimentaires », Gérontologie et société, $\mathrm{n}^{\circ}$ 134, 2010, pp. 31-42.

CARDON Philippe, « Manger en vieillissant pose-t-il vraiment problème ? Veuvage et transformations de l'alimentation des personnes âgées ", Lien Social et Politiques, n²62, 2009a, pp. 85-95.

CARDON Philippe, «Retraite et alimentation : les effets de la mobilité », Recherches familiales, $\mathrm{n}^{\circ} 6$, 2009b, pp. 105-115.

CARDON Philippe et GOJARD Séverine, « Les personnes âgées face à la dépendance culinaire : entre délégation et remplacement », Retraite et société, n 56, 2009, pp. 169-193.

CARRASCO Noelia, «Desarrollos de la antropología de la alimentación en América Latina : hacia el estudio de los problemas alimentarios contemporáneos ", Estudios Sociales, vol. 16, n 30, 2007, pp. 79-102.

CASCUDO Luis da Camara, Historia da alimentaçao no Brasil, Sao Paulo, Universidade de Sao Paolo, 1983.

CHAVES Mônica, «La restauration hors foyer dans l'État brésilien de Minas Gerais », Journal des Anthropologues, $\mathrm{n}^{\circ}$ 106-107, 2006, pp. 189-204.

COLE Nicki, « Global capitalism organizing knowledge of race, gender and class : The case of socially responsible coffee », Race, Gender and Class, vol. 15, n 1-2, pp. 170-187.

CONTRERAS Jesus y GRACIA Mabel, Antropología y Cultura. Perspectivas antropológicas, España, Ariel, 2005. 
CORBEAU Jean-Pierre et POULAIN Jean-Pierre, Penser l'alimentation. Entre imaginaire et rationalité, Paris, Privat, 2002.

CORBEAU Jean-Pierre (coord.), Cuisine, alimentation, métissage, Paris, Bastidiana, 2000.

COTTOM Bolfy, « El patrimonio cultural como problema interdisciplinario », Red Patrimonio : Revista digital de estudios en patrimonio cultural, 2007a.

COTTOM Bolfy, « Diversidad y enfoques del patrimonio cultural », Cuadernos del Patrimonio Cultural y Turismo, $\mathrm{n}^{\circ} 8,2004$.

DONNAT Olivier et TOLILA Paul (eds.), Le(s) public(s) de la culture, Paris, Presses de Sciences Po, 2003.

DONNAT Olivier, Les français face à la culture. De l'exclusion à l'éclectisme, Paris, La Découverte, coll. « Textes à l'appui », 1994.

DOUGLAS Mary, « Les structures du culinaire », Communications, vol. 31, 1979, pp. 145-70.

DURKHEIM Émile et MAUSS Marcel, « De quelques formes primitives de classification.

Contribution à l'étude des représentations collectives ", Anneé sociologique, n 6, 1903.

DUVAL Julien, « Distinction studies », Actes de la recherche en sciences sociales, $n^{\circ} 181-182,2010$, pp. 146-156.

ELOY Florence et PALHETA Ugo, «Cultures juvéniles et enseignement musical au collège », Revue française de pédagogie, $\mathrm{n}^{\circ}$ 163, 01 juin, 2008. Disponible en ligne : http://rfp.revues.org/957.

ELOY Florence, Apprendre à écouter la musique. Culture légitime, culture scolaire et cultures juvéniles, Paris, EHESS, thèse, (dir. D. Pasquier), 2012.

ESPEITX Elena, « Los sentidos del patrimonio alimentario en el sur de Europa » in Marcelo ALVAREZ y Francisco-Xavier MEDINA (eds.), Identidades en el plato. El patrimonio cultural alimentario entre Europa y América, Barcelona, Icaria, 2008, pp. 45-62.

FERNANDEZ-ARMESTO Felipe, A comida : uma historia, Rio de Janeiro, Record, 2004.

FISCHLER Claude et MASSON Estelle, Manger. Français, Européens et Américains face à

l'alimentation, Paris, Odile Jacob, 2007.

FLANDRIN Jean-Louis et MONTANARI Massimo, Histoire de l'alimentation, Paris, Fayard, 1996.

FLORES Jesus, Breve historia de la comida mexicana, México, De Bolsillo, 2003.

FLORESCANO Enrique (coord.), El patrimonio cultural de México, México, FCE, 1993.

FREYRE Gilberto, Casa grande e senzala. Formação da família brasileira sob o regime de economia patriarcal, Rio de Janeiro, Maia e Schmidt, 1933.

GABACCIA Donna, We are what we eat. Ethnic Food and the Making of the Americans, Boston, Harvard Univeristy Press, 1998.

GARNSEY Peter, Food and Society in Classical Antiquity, Cambridge, 1999.

GARCIA-GARZA Domingo, « Una etnografía económica de los tacos callejeros en México. El caso de Monterrey », Estudios Sociales, vol. 19, n 37, 2011, pp. 32-63.

GARCIA-GARZA Domingo, « Prácticas alimenticias y clasificación social. ¿Los tacos son un alimento 'popular' ?», Civitas, vol. 10, n 3, 2010, pp. 430-449.

GARCIA-GARZA Domingo, L'entreprenariat informel. Le cas des marchands de tacos à Monterrey (Mexique), Paris, EHESS, thèse, (dir. R. Lenoir), 2009. 
GOJARD Séverine, Le métier de mère, Paris, La Dispute, coll. « Corps santé société », 2010.

GOODY Jack, Cooking, Cuisine and Class, London, Cambridge University Press, 1982.

GRIGNON Claude et GRIGNON Christine, «Styles d'alimentation et goûts populaires », Revue française de sociologie, 1980, vol. 21, pp. 531-569.

HERPIN Nicolas et VERGER Daniel, Consommation et modes de vie en France. Une approche économique et sociologique sur un demi-siècle, Paris, La Découverte coll. « Grands Repères », 2008.

HIRAI Shinji, Economía política de la nostalgia : un estudio sobre la transformación del paisaje urbano en la migración transnacional entre México y Estados Unidos, México, UAM/Juan Pablo Editor, 2009.

LAMONT Michèle, Money, Morals, and Manners: The Culture of the French and the American UpperMiddle Class, Chicago, University of Chicago Press, 1992.

LEVI-STRASS Claude, Mythologiques, Paris, Plon, (4 volumes), 1964.

LONG-SOLIS Janet y VARGAS Luis, (eds.), Food Culture in Mexico, Westport/London, Greenwood Press, coll. « Food Culture Around the World », 2005.

MONCUSí Albert y SANTAMARINA Beatriz, « Bueno para comer, bueno para patrimonializar. La propuesta de la cocina mexicana como Patrimonio Inmaterial de la Humanidad » in Marcelo ÁLVAREZ y Francisco-Xavier MEDINA (eds.), Identidades en el plato. El patrimonio cultural alimentario entre América y Europa, Barcelona, Icaria, 2008, pp. 127-142.

MAGALHãES (de) Sônia M., A Mesa de Mariana : Produção e consumo de alimentos em Minas Gerais (1750-1850), São Paulo, Annablume/Fapesp, 2004.

MARTINEZ Irma y VILLEZCA Pedro, « La alimentacion en México. Un estudio a partir de la encuesta nacional de ingresos y gastos de los hogares ", Notas : Revista de información y análisis (INEGI), 2003, pp. 26-37.

MINTZ Sidney, Tasting food, Tasting Freedom : Excursions into Eating, Culture, and the Past, Boston, Beacon Press, 1996.

MINTZ Sidney and DUBOIS Christine, " The Anthropology of Food and Eating ", Annual Review of Anthropology, $\mathrm{n}^{\circ}$ 31, 2002, pp. 99-119.

ORTIZ Ana Silvia, VÁZQUEZ Verónica y MONTES Margarita, « La alimentación en México. Enfoques y visión de futuro », Estudios Sociales, vol. 13, n² 25, 2005, pp. 8-34.

OSEGUERA David, « La comida. Lugar de encuentro entre disciplinas científicas », Estudios sobre las culturas contemporáneas, vol. 7, n 13, 2001, pp. 141-151.

PETERSON Richard, « Understanding audience segmentation : from elite and mass to omnivore and univore ", Poetics, $n^{\circ} 32,1992$, p. 169-194.

PETERSON Roger et KERN R.M., « Changing highbrow taste : from snob to omnivore », American Sociological Review, vol. 61, n 5, 1996, pp. 900-907.

PILCHER Jeffrey M., ; Que vivan los tamales! Food and the Making of the Mexican Identity, Albuquerque, University of Mexico Press, 1998.

PILCHER Jeffrey M., Planet Taco : A Global History of Mexican Food, New York, Oxford University Press, 2012.

POULAIN Jean-Pierre (dir.), Dictionnaire des cultures alimentaires, Paris, PUF, 2012. 
POULAIN Jean-Pierre, «French gastronomie, french gastronomies » in Dara GOLDSTEIN and Kathrin MERKELE (eds.), Culinary cultures of Europe. Identity, Diversity and Dialogue, Strasbourg, Council of Europe Publishing, 2005, pp. 157-170.

POULAIN Jean-Pierre, Sociologies de l'alimentation. Les mangeurs et l'espace social alimentaire, Paris, PUF, coll. «Sciences sociales et sociétés », 2002.

POULAIN Jean-Pierre, Manger aujourd'hui : attitudes normes et pratiques, Toulouse, Privat, 2001.

POULAIN Jean-Pierre, « Les patrimoines gastronomiques et leurs valorisations touristiques » in Rachid AMIROU et Philippe BACHIMON, Le tourisme local, une culture de l'exotisme, L'Harmattan, 2000, pp. 157-183.

POULAIN Jean-Pierre, «Le gout du terroir à l'heure de l'Europe », Ethnologie française, vol. 27, 1997, pp. 18-26.

RITZER George, The MacDonaldization of Society. An Investigation Into the Changing Character of Contemporary Social Life, London, Sage, 1993.

STANZIANI Alessandro, Histoire de la qualité alimentaire XIX ${ }^{e}-X X^{e}$ siècles, Paris, Seuil, coll. « Liber », 2005.

SUREMAIN (de) Charles-Édouard et KATZ Esther, « Modelos alimentarios y recomposiciones sociales en América Latina », Anthrolpology of Food, 20 déc., 2009. Disponible en ligne : http:// aof.revues.org/6432.

SWIDLER Ann, Talk of Love : How Culture Matters, Chicago, University of Chicago Press, 2001.

TAYLOR Stephen, SMITH Shenna and LYON Phil, « McDonalization and consumer choice in the future : an illusion or the next marketing Revolution? » in Mark ALFINO, John S. CAPUTO and Robert WYNYARD (eds.), Mcdonaldization Revisited. Critical Essays on Consumer Culture, Westport/ London, Prager Publishers, 1998, pp. 105-119.

THIESSE Anne-Marie, La création des identités nationales. Europe XVII ${ }^{e}-X X^{e}$ siècles, Paris, Seuil, 1999.

TILLY Charles, «Contentious repertoire in Great Britain, 1758-1834 », Social Science History, vol. $17, \mathrm{n}^{\circ} 20$, pp. 253-280.

TINKER Irene, Street Food. Urban Food and Employment in Developing Countries, Oxford, Oxford University Press, 1997.

TORRES Felipe y TRAPAGA Yolanda (eds.), La alimentación de los Mexicanos en la alborada del tercer milenio, México, UNAM, 2001.

VARGAS Luis A., « Antropología y alimentación », Antropológicas, n 7, 1993, pp. 22-23.

ZAPATA Sergio, «Patrimonialización de la gastronomía peruana y planteamiento de un proyecto de desarrollo » in Marcelo ÁLVAREZ y Francisco-Xavier MEDINA (eds.), Identidades en el plato. El patrimonio cultural alimentario entre América y Europa, Barcelona, Icaria, 2008, pp. 153-174.

\section{AUTEURS}

\section{PHILIPPE CARDON}

Maître de conférences en sociologie, Université Lille 3. Chercheur au laboratoire Ceries (laboratoire de recherche individus-épreuves et société) / Université Lille 3. Chercheur associé au laboratoire ALISS (Alimentation et sciences sociales)/INRA.

Philippe.Cardon@ivry.inra.fr 


\section{DOMINGO GARCIA-GARZA}

Maître de conférences en civilisation latino-américaine (Université Charles de Gaulle). Chercheur au CECILLE (Lille 3) et chercheur associé au CESSP (EHESS/Paris 1/CNRS).

domingo.garciagarza@univ-lille3.fr 